\title{
LUXURIA MAURETANIAE. ¿LA EXPLOTACIÓN DE LOS PRODUCTOS DE LUJO COMO CAUSA DE LA CONQUISTA?
}

\author{
LLUIS PONS PUJOL \\ Universitat de Barcelona \\ llpons@ub.edu \\ ORCID: 0000-0002-5396-8352
}

\section{RESUMEN}

Se ha propuesto que la causa de la conquista del reino de Mauritania en época de Calígula fuera el deseo de explotar algunos recursos naturales, considerados de lujo. Analizamos los datos aportados por las fuentes literarias y epigráficas. Concluimos que la explotación de estos recursos, por si sola, no puede considerarse la causa de la conquista.

PALABRAS CLAVE: Mauritania, Calígula, maderas preciosas, púrpura, marfil.

\section{THE EXPLOITATION OF LUXURY PRODUCTS AS A CAUSE OF THE CONQUEST?}

\section{ABSTRACT}

It has been proposed that the cause of the conquest of the kingdom of Mauritania in Caligula's time was the desire to exploit some natural resources, considered luxury. We analyze the data provided by literary and epigraphic sources. We conclude that the exploitation of these resources, by itself, cannot be considered the cause of the conquest.

KEYWORDS: Mauritania, Caligula, precious woods, purple, ivory.

\section{Hipótesis SObRe LA CONQUISTA DEL REINO DE MAURITANIA}

El reino de Mauritania formaba parte de facto de los territorios controlados por Roma, como mínimo desde Augusto, que dudaba entre convertirlo en provincia o en un estado vasallo. ${ }^{1}$ En 25 a.C. decidió colocar en su trono a Juba II y por tanto debemos considerarlo como uno de los reges socii et amici populi romani (Cimma 1976). A inicios del año 40 d.C. Calígula decidió convertir en provincia este reino y ordenó asesinar a su primo el rey Ptolomeo. ${ }^{2}$ Esto generó la reacción lógica de

${ }^{1}$ Dión Casio, LIX, 43, 7; Coltelloni-Trannoy 1997: 79-80; Amela 2012; Bernard 2018: 197-244.

2 Según Dión Casio, LIX, 25, 1 y LX 8, 6, a principios del año 40 d.C., encontrándose Calígula en Lugdunum, ordenó asesinar a Ptolomeo por su riqueza, indicando además que Claudio, debido a la insistencia de sus libertos, aceptó el triunfo sobre la Guerra de Mauretania aunque ésta había ya terminado cuando él accedió al trono; Suetonio, Caligula, XXXV, 1 indica que Calígula mandó llamar a Ptolomeo y lo colmó de honores, pero lo mandó asesinar de improvisto por la envidia que le causó que el pueblo se fijara en una espléndida toga púrpura que vestía el monarca (Guastella 1994: 11-57). Por otro lado, Séneca, De tranquillitate animi, XI, 1, indica que en esa época vio a Ptolomeo en la cárcel en Roma. Las emisiones monetales de Ptolomeo finalizan sin duda en el 39 d.C. (Mazard 1955: no 496). 
algunos súbditos del monarca mauritano y una guerra de conquista de dos años (Pons 1997, 2008, 2014). La provincia imperial de Mauretania fue creada inmediatamente ${ }^{3}$ y después de la guerra, fue dividida en las dos provincias procuratorias de Mauretania Tingitana y Mauretania Caesariensis. ¿Por qué convirtió en provincia un reino vasallo que no había generado nunca problemas a Roma?

Se han propuesto distintas hipótesis para explicar este hecho, que pueden ser agrupadas bajo dos grandes epígrafes, hipótesis de raíz política e hipótesis de raíz económica. Veamos, en primer lugar, las hipótesis que se centran en considerar la coyuntura política del momento, que son las que han generado más adhesión. Para Carcopino (1943: 194) la causa se halla en el carácter desequilibrado y megalo-maníaco del emperador, al que debemos sumar su necesidad de dinero. Al ser parientes y no tener descendencia Ptolomeo, si éste moría sería el emperador quien recibiría su herencia. En su concepción de los hechos, Calígula tomó una decisión rápida, tomando como excusa la toga púrpura. Para Hoffmann (1959) la causa sería un conflicto religioso entre Ptolomeo, Gran sacerdote de Isis, y Calígula que deseaba obtener este cargo. Su razonamiento se basaría en la interpretación de la toga de púrpura como la vestimenta propia de los grandes sacerdotes de Isis. Para Kotula (1964) la causa se hallaría en las luchas e intrigas entre los libertos de los dos monarcas. Para Faur (1973: 249-253) la hipótesis isíaca no sería sostenible; la causa real estaría, según este autor (1973: 260-264), en tendencias independentistas y filosenatoriales del monarca mauritano que pueden ser detectadas en las emisiones de monedas de oro de los años 38-39 d.C., Leveau (1984a, 1984b: 1316), sin aceptar la tesis suetoniana de la locura del emperador, considera que no deben buscarse motivos políticos a la muerte de Ptolomeo. Para él, Calígula veía en su pariente solamente un miembro más de la aristocracia imperial y fue asesinado como tantos otros durante su principado. Ghazi ben Maissa (1995) considera que las causas son esencialmente políticas: Ptolomeo era un claro capax imperii por sus vínculos familiares con los lágidas y con Marco Antonio; al estar relacionado con la conspiración de Gaetulicus, Calígula decidió eliminar este adversario. Para Coltelloni-Trannoy (1988-1989, 1997: 56-59, 177-181), la hipótesis isíaca no sería aceptable, pero tampoco la de las emisiones en oro; para esta autora la causa estaría en la implicación de Ptolomeo en la conspiración de Gaetulicus. Lenoir (2001) cree acertadas las críticas de Faur y Coltelloni-Trannoy sobre la hipótesis isíaca, pero no está de acuerdo en la hipótesis política ya que considera que la intervención de Ptolomeo en la conspiración de Gaetulicus es puramente hipotética y poco plausible. Para Lenoir, Calígula ordenó el asesinato de Ptolomeo por un conjunto de factores: el carácter psicópata del emperador, la

\footnotetext{
${ }^{3}$ Di Vita justifica la fecha del 40 d.C. para el asesinato del rey y el nacimiento de la nueva provincia mediante la dedicatoria de los horrea de Tubusuctu (CIL VIII, 8836; Di Vita 1992: 844-847, 1994). Sobre los gobernadores de la provincia Mauretania, cf., Pons 2020.
} 
supuesta participación de Ptolomeo en la conspiración de Gaetulicus, la rivalidad que los dos personajes pudieran haber tenido por considerarse herederos de la tradición antoniana, y el deseo del emperador de apropiarse de las riquezas del monarca. La hipótesis más reciente es la de Schettino (2003), para quien se trata solamente de un paso más en la necesaria e inevitable provincialización de los estados vasallos.

En cuanto a las hipótesis que privilegian los motivos económicos, Rostovzev (1933 [1992]: 372-373) considera fundamental la necesidad de nuevas tierras para asentar a colonos itálicos. Para Sigman (1977) la causa es estratégica: la defensa del Estrecho y de las provincias norteafricanas productoras de cereales. Para Gozalbes (1988, 1997: 177-178), la causa estaría en la voluntad de Roma de explotar los recursos naturales mauritanos (púrpura, madera, animales salvajes, entre otros) y lo justificaría indicando que Plinio, treinta años después de la conquista, en sus comentarios sobre la Tingitana, no se refiere a su valor agrícola sino a los productos suntuarios anteriormente mencionados.

Analicemos los datos referentes a estos productos suntuarios que interesaban tanto a la élite romana por ser expresión de riqueza personal y reflejo de su dominio del Imperio, ${ }^{4}$ el lujo procedente de Mauretania: luxuria mauretaniae.

\section{LA MADERA}

No existe ningún trabajo de conjunto sobre la explotación de la gran riqueza vegetal y forestal del reino mauritano y de la posterior provincia Mauretania Tingitana a pesar de que las fuentes greco-romanas aluden a ella en diversas ocasiones. ${ }^{5}$ Besnier (1906) fue el primero en tratar el tema, comentando algunas generalidades. Carcopino (1943: 43) insistió especialmente sobre la rapacidad romana en relación con la madera de cidro. Finalmente, Jodin (1970a), recogió algunas fuentes sobre este tema.

La madera más preciada era la del árbol llamado citrus en latín y thyon o thya en griego, que ha sido traducido erróneamente al castellano como cedro o como limonero. La confusión proviene de la propia denominación antigua, utilizada indistintamente por las fuentes para designar dos especies distintas de árboles, el cedro de Oriente y la tuya del mediterráneo occidental, así como

${ }_{4}^{4}$ «La description précise et constante de la provenance de ces produits souvent rare et chers, que la société romaine toute entière convoite, montre, en même temps que l'importance du commerce et le goût de l'exotisme, l'assimilation entre la puissance impérialiste de Rome et la possession de produits de luxe. C'est la puissance de Rome sur l'Empire et, au-delà, sur l'ensemble du monde connu, qui est implicite dans les manteaux tyriens, les tables en cédratier de Libye, les émeraudes de Scythie, les jeunes esclaves grecs et égyptiens, les soieries de Cos ou de Chine, les tables de Maurétanie aux pieds d'ivoire ... pour ne citer que quelques exemples», Dubois-Pelerin 2008: 241-242. Cf. también Dalby 2000.

${ }^{5}$ Hdt. 4, 191 y 198; Strab. 2. 5. 33, 17. 3. 10-15; Luc. Fars. 9. 426-429; Stat. Silv. 3. 3; Mela 1. 5, 3. 10; Plin. Nat. 5. 6, 13.29-30; Solin. 25; Ov. Met. 4. 658, 4. 779. 
algunos cítricos (Pagnoux 2017). Aunque se alude comúnmente en la bibliografía a esta especie como cidro, su nombre más apropiado en castellano (López González 2001: 241-244) sería sabina de Cartagena (Tetraclinis articulata).

Varios autores permiten constatar la importancia de esta actividad en la Tingitana. Lucano (Fars. 9. 426-429) comenta exageradamente que los mauri tenían los árboles como única fuente de riqueza y cómo los romanos llegaron hasta su país para buscar la madera con la que construir mesas lujosas. Estacio (Silv. 3.3), tratando de las grandes riquezas de cada territorio, alude a los cidros de Mauritania. Estrabón (17. 3. 4) indica que de los bosques tingitanos procedían las mesas de madera de una sola pieza, con variados colores y de gran tamaño. Horacio (Odas 4. 1) habla de los artesonados de madera de cidro de los templos de Roma.

Pero la fuente más extensa es Plinio (Nat. 13.29-30). Según este autor, la belleza de esta madera radicaba en que mostraba unas vetas similares al mármol y con ella se realizaban mesas de una sola pieza muy apreciadas por la élite romana. Los diferentes tipos de venas servían para distinguir diferentes tipos de madera: la que recordaba la piel de un tigre era tigrinum apellatur, la que recordaba la piel de pantera era pantherinae vocantur y apiatas vocant a las que recordaban las hojas del apio. El color también era un factor para tener en cuenta, siendo las más apreciadas las de color vino: hic maxime mulsi placet (Nat. 13.30).

Según Plinio (Nat. 13.29.1), la posesión de estas mesas generaba en los hombres la misma pasión que la que ellos reprochaban a sus esposas por las perlas (Pérez 2014, 2019): mensarum insania, quas feminae viris contra margaritas regerunt. Los altísimos precios a que llegaron a venderse mesas de esta madera tenían la causa en varios factores: en primer lugar, en que la de más calidad solamente se encontraba en el Atlas mauritano: ${ }^{6}$ en segundo lugar, en que, hasta el principado de Nerón, su explotación fue discreta; $y$, en tercer lugar, en que la demanda era enorme. Se llegó a pagar más de un millón de sestercios por unidad y la más cara que tenemos documentada, destruida en el incendio de Roma del 64 d.C., costó 1.300 .000 sestercios.

Gozalbes (1997: 181) cree que la explotación forestal habría podido ser un monopolio de la monarquía mauritana que vendería el producto a los negotiatores romanos, asociados en una corporación de citriarii, ${ }^{7}$ y éstos, a su vez, al rico comprador. Pero no lo justifica, así como tampoco expone qué pudiera haber pasado con este supuesto monopolio en el momento de la anexión del reino mauritano como provincia romana. De manera que no tenemos ninguna referencia que pudiera indicar que los monarcas mauritanos, y los emperadores romanos después, controlasen la explotación de los bosques tingitanos.

La evolución de la explotación es la siguiente: se inició con la dominación cartaginesa pero no fue hasta el reinado de Juba II cuando se organizó

\footnotetext{
${ }^{6}$ Sobre el Atlas en la antigüedad, $c f$. Enc. Ber. sv. «Atlas»; Cheddad 2002: 2163-2176.

${ }^{7}$ CIL VI, 33855.
} 
sistemáticamente (Strab. 17. 3. 4-6). Desde la conquista de la provincia (40 d.C.) hasta el principado de Nerón se explotó pero a un nivel bajo. Podemos llegar a esta conclusión a partir de los precios extraordinariamente altos que tenían en este momento las mesas de citrus. En tiempos de Plinio el Joven, la madera de más calidad, que era la producida en el Mons Ancorarius, ya estaba agotada (Nat. 13. 29), debiéndose combinar en la construcción de la mesa el citrus con el marfil: el primero se utilizaba en la parte superior de la mesa y el segundo en las patas. El marfil utilizado era también de origen mauritano y, como consecuencia de esta demanda, aumentó de precio (Mart. Ep. 2. 43; 9. 22; 10. 98; 14. 89, 90 - 91). A principios del s. II d.C. se produjo un descenso de su exportación debido al agotamiento de los bosques y a la saturación del mercado de Roma. Tertuliano todavía critica, a finales del s. III d.C., el precio de las mesas de citrus (De pallio, 7). ${ }^{8}$ Se ha propuesto que Mogador fuera un commercium romano dedicado a la exportación de la madera de este árbol, abundante en sus alrededores (Delgado 2011).

La madera de cidro fue, por tanto, uno de los principales recursos que los romanos buscaron en el territorio del reino mauritano, acaparándola de tal forma que se destruyeron los bosques de esta especie.

\section{EL MARFIL}

El marfil más apreciado por los romanos era el que procedía de la India, ya que los colmillos de sus elefantes eran más grandes. El procedente de la Tingitana, como el del resto de las provincias norteafricanas, presentaba la ventaja de encontrarse en una zona relativamente cercana, hecho que abarataba el producto. Hasta su definitiva desaparición, en el s. III d.C., los elefantes del Marruecos antiguo proporcionaron cantidades ingentes de marfil, siendo este producto una de las exportaciones mauritanas mejor consideradas en Roma por su carácter suntuario.

Según diversos autores, el comercio del marfil del Norte de África hacia Europa se daba ya desde el Eneolítico cuando se procedía a intercambiarlo por cerámicas campaniformes u objetos de metal (Jodin 1970b, Harrison y Gilman 1977, Souville 1983). La presencia de elefantes en este momento, así como su captura está probada mediante el hallazgo de restos óseos, así como de pinturas rupestres (Antoine 1931, Jodin 1970b: 54-62). Estudios relativos al periodo cuaternario en Marruecos demuestran que el elefante cazado por los romanos para la obtención de sus colmillos, el Elephas africanus, era un animal de menor tamaño que los que existieron con anterioridad (Jodin 1970b: 51, Beaudet 1971).

En cuanto a las fuentes greco-romanas sobre el elefante, el primero en estudiar ampliamente este tema a nivel de todo el Norte de Africa fue Gsell

\footnotetext{
${ }^{8}$ Cf. Carcopino 1943: 41, Gozalbes 1995: 186.
} 
(1920). ${ }^{9}$ Besnier (1906) lo estudió muy brevemente y solo a nivel tingitano. Posteriormente se interesaron por él, Jodin (1970b) y Gozalbes (1988), relacionando las informaciones aportadas por las fuentes y las evidencias arqueológicas.

El marfil mauritano fue explotado ya en época cartaginesa. En primer lugar, el Periplo de Hannón menciona la presencia abundante del elefante más allá de las Columnas de Hércules (Peripl. 4), habiendo sido identificado (Gozalbes 1997: 189) este lugar como la zona pantanosa existente en Sidi Kacem, al Sur del cabo Achakar (Tánger). Por otro lado, el Periplo de Scylax indica que uno de los principales objetos de comercio de los cartagineses en el litoral atlántico africano era el marfil, añadiendo que los indígenas lo utilizaban ampliamente en su vida cotidiana (Scyl. 112). En tercer lugar, Gozalbes piensa que la referencia de Herodoto sobre el comercio mudo debe relacionarse con el marfil y no con el oro, inexistente en el litoral mauritano (Scyl. 112; Gozalbes 1997: 189).

En cuanto al comercio de este producto con Roma, empezó a exportarse bajo el reino de Bogud y fue con Juba II cuando este comercio aumentó significativamente (Prop. 2. 31. 12; Ov. Pont. 4. 9. 28).

Las fuentes coincidían en indicar la abundancia de elefantes en la Tingitana, cazándose tanto para la obtención de sus defensas (Ov. Pont. 4. 9. 28) como para ser enviados a Roma vivos, donde eran utilizados en los espectáculos públicos (Prop. 2. 31. 12). Estrabón, menciona que abundaban en Mauritania (Strab. 17. 3. 4; Biffi 1999: 382) hasta el punto que un monte recibía el nombre ' $\mathrm{E} \lambda \varepsilon \varphi \alpha \varsigma$ (17.3. $6)^{10}$ y eran cazados por los indígenas ${ }^{11}$ para, entre otros motivos, confeccionar escudos con su piel (17. 3. 7). También Pomponio Mela alude a estos escudos, cuando menciona la existencia en los alrededores de Tingi de un gran escudo de piel de elefante que la población atribuía al fundador mítico de la ciudad, el gigante Anteo (1.5). ${ }^{12}$ Por otro lado, Estrabón critica por fantasiosas las virtudes de la inteligencia de los elefantes del Norte de la Mauritania que el escritor de época de César, Tanusio Gémino, ofrece de ellos: combatían el fuego, mientras los demás animales lo temían, debido a que entendían que éste devoraba las plantas con las que se alimentaban; también luchaban contra los hombres en

\footnotetext{
${ }^{9}$ Cf. también Keller 1904, 1909, 1914.

10 «No deja de ser cierto que, como se ha indicado en ocasiones, desde algunos puntos de vista la silueta del Yebel Fahies y el Yebel Musa forman la figura petrificada de un elefante; no obstante, pensamos que junto a este factor el topónimo viene a indicar la existencia de numerosos elefantes en toda esta zona», Gozalbes 1988: 5; «E $\lambda \varepsilon \varphi \alpha \varsigma$ avrà avuto attinenza o con la particolare conformazione della costa o con la presunta presenza di elefanti nell'area», Biffi 1999: 385.

11 Sobre los de la zona de Septem Frates: «Junto a ella, en el pasillo de Anyera, la epigrafía documenta el asentamiento del pueblo de los Masaisuli (I.A.M.lat., no 52). Muy probablemente eran estos indígenas los que efectuaban la caza...», Gozalbes 1997: 190. Esta afirmación nos parece temeraria. Se trataría de un pueblo más de la zona.

${ }^{12}$ Cf. sobre Mela y la Tingitana, Gozalbes 1995. Cf. sobre Anteo, Enc. Ber. sv. «Antée».
} 
manada y cuando eran heridos se arrodillaban delante de ellos pidiendo perdón (Strab. 17. 3. 8). ${ }^{13}$

La conquista romana de la Tingitana supuso un punto de inflexión en la explotación del marfil de esta zona ya que fue uno de los principales productos que los romanos explotaron en la nueva provincia con el objetivo de dedicarlo a la elaboración de productos suntuarios (Plin. Nat. 5. 12). De marfil eran joyas de todo tipo, así como las llamadas estatuas crisoelefantinas (Plin. Nat. 8. 24). Sin olvidar las patas de las mesas de cidro que, habiéndose ya agotado en parte este producto, como hemos señalado, se completaba con marfil dotándolas, a ser posible, de un carácter todavía más lujoso (Dubois-Pelerin 2008: 225-228).

Plinio es la fuente principal para este periodo. Nos informa detalladamente de la existencia de este animal en todo el Norte de África y que siendo típicamente africano (Plin. Nat. 8. 24), era abundantemente cazado (Nat, 8. 9; 8. 21). Menciona concretamente que había muchos de ellos en las montañas de Gomara y Yebala hasta Septem Frates (Nat. 5. 18), en el bosque de la Mamora y regiones más al Sur (Nat. 5. 5). Junto con estos datos, también recoge fantasías basadas en la obra de Juba II, ${ }^{14}$ según el cual los elefantes se purificaban solemnemente en el río Amilo y que adoraban al Sol naciente y a la Luna nueva, o bien que, dando pruebas de su gran inteligencia, rompían sus defensas contra un árbol al verse acorralados por el hombre pues entendían qué era lo que éste deseaba de ellos (Nat. 8. 1, 8. 8-10). Insiste también en destacar la abundancia de elefantes en Septem y su caza sistemática el africano Julio Solino que escribió en el s. III d.C. basándose en textos de autores de mediados del s. I d.C., sobre todo Plinio (Solino. Coll. rer. mem. 24). Asimismo, debemos tener en cuenta las afirmaciones en el mismo sentido del africano del s. IV d.C. Marciano Capella, igualmente fundamentadas en autores de siglos anteriores (Marciano Capella, De Nup. Phil. 6. 668). De estas fuentes se puede extraer la conclusión que Septem tenía en la caza, explotación y exportación del marfil en el s. I d.C. una importante fuente de ingresos (Gozalbes 1988: 11, 1997: 190-191).

No tenemos ningún dato acerca de si el marfil era trabajado en la Tingitana, lo que supondría toda una floreciente industria en esta zona, o si por el contrario se enviaba a Roma en bruto. Para el caso concreto de las mesas de cidro con patas de marfil, se ha propuesto - sin ofrecer datos concretos - que la manufactura se realizara en la propia Tingitana (Gozalbes 1997: 192).

Tampoco tenemos datos concretos sobre los puertos desde los que se exportó. Se supone que debieron ser los más cercanos a las zonas en que los

${ }^{13}$ Cf. Biffi 1999: 390.

${ }^{14}$ El estudio de Juba II sobre los elefantes responde a la curiosidad que de ellos tenía el Mundo Romano, dado que cualquier objeto de marfil era relacionado con ellos. Pero se trata de investigaciones superficiales, a partir de la lectura de obras anteriores, sin un conocimiento directo de las costumbres de estos animales. Plinio advierte que Juba considera las defensas del elefante como cuernos, cuando Herodoto ya los había identificado como dientes (Plin. Nat. 8. 7; Herodor. 3. 97). Cf. Sirago 1996. 
elefantes abundaban: Septem (Gozalbes 1997: 191), que drenaría las piezas cazadas en la zona entre el Yebel Mussa y el Istmo ceutí; Tamuda, que a pesar de no tener puerto, centralizaría las piezas cazadas en el Rif occidental y Tetuán; Tingi, naturalmente como principal puerto de la provincia y Sala, que absorbería las piezas llegadas desde el Atlas.

Seguramente fueron comerciantes romanos los que lo exportaron, aunque no conocemos ninguno de sus nombres. Ni se les honró en la Tingitana con un monumento como el que el ordo de Leptis Magna dedicó a un Porfirio, amator patriae et civium suorum, que se ganó el honor al donar civibus suis quattor feras dentatas vivas, es decir, elefantes (IRT, 603). Se trataría de un exportador de animales del Norte de África, como indica su donación y las representaciones de naves que el ordo mandó esculpir en las pilastras de su monumento, que sostenía una biga (Rostovzev 1933: 86, tav. 66.2).

La depredación de este recurso llevó consigo su rápido agotamiento. Según las fuentes, durante los flavios ya era raro que se consiguieran piezas de gran tamaño en esta zona, refugiándose los elefantes en el interior de los bosques (Nat. 8. 4, 8. 10).

Durante el s. II d.C. su caza continuó a un ritmo considerable, como nos documenta Timoteo de Gaza ${ }^{15}$ y sobre todo Eliano que ofrece una descripción de sus costumbres llena de mitos: la existencia de un cementerio de elefantes cerca del Atlas, la prohibición de los habitantes del Atlas de cazarlos, su proverbial inteligencia, entre otros (Ael. VH. 7. 2, 9. 8, 14. 5-6). ${ }^{16}$ De tal modo que en un momento indeterminado del s. III d.C. fueron totalmente aniquilados de la Tingitana. Se ha propuesto que a principios de este siglo no hubieran desaparecido todavía, dado que en una remisión de impuestos concedida por Caracalla en 216 d.C. a la ciudad de Banasa se citan a las caelestium fertilibus animalium, interpretados como elefantes destinados al emperador (I.A.M.lat. 100, 1. 14; Guey 1947).

Muy a principios del s. IV d.C., el Edictum de pretiis no incluye ya a los elefantes entre los animales africanos que fueran susceptibles de ser destinados a los juegos de anfiteatro (Lassère 1995: 40). También en el s. IV d.C., Themistios documenta la extinción del elefante en todo el Norte de África (Them. Or. 10). Y, tres siglos más tarde, Isidoro de Sevilla, utilizando una fuente de la segunda mitad del s. III d.C. indica que, en el pasado, estos animales habían vivido en el Norte de África, pero que en su época solamente vivían en la India (Isid. Et. 12. 2. 16, 14. 5. 12).

En el s. XII d.C. un autor árabe menciona la presencia de elefantes en la región de Marrakech y, sobre todo en el Sur de Marruecos (región de Sus), pero quizás utiliza fuentes del s. VIII d.C. (Siraj 1995: 199, 207).

\footnotetext{
15 apud Gozalbes 1997: 192.

16 Sobre el Atlas en la antigüedad, cf. Enc. Ber. sv. «Atlas»; Hamdoume 2009.
} 
Debemos considerar al marfil, en consecuencia, otro de los productos de lujo que los romanos buscaron en el reino de Mauritania y en las provincias Tingitana y Caesariensis, siendo ampliamente exportado hacia Roma, donde las mesas $u$ objetos de este material se pusieron de moda.

\section{LOS ESCLAVOS}

La Tingitana es una provincia en la que no se detecta una fuerte presencia de mano de obra esclava, pero sí fue una zona exportadora de esclavos. Puede considerarse que la consecuencia directa para los indígenas de diversos enfrentamientos con Roma, como la llamada revuelta de Aedemón, la guerra de conquista, y las luchas posteriores durante los s. I y II d.C., fuera la reducción a la esclavitud de los prisioneros (De Martino 1985: 335).

Roma actuaba con los esclavos como con los otros productos del territorio, es decir, los explotaba y exportaba según sus necesidades, sin tener en cuenta las de la provincia. En este sentido, podemos pensar que, dejando de lado el evidente factor de sangrado demográfico que suponía la exportación de esclavos, no implicó una alteración de la economía provincial por varios motivos. En primer lugar, la economía provincial no estaba basada en el elemento esclavo. En segundo lugar, en el conjunto del Alto Imperio no debió exportarse una cantidad muy grande, pues los conflictos graves se dieron en momentos puntuales. Y en tercer lugar, la reducción a la esclavitud implicó sobre todo a poblaciones nómadas que, al vivir la mayor parte del año fuera del territorio realmente controlado por Roma, no afectaban a la globalidad de la economía provincial.

No es hasta la época de Juba II y Ptolomeo cuando se conocen las primeras inscripciones relativas a esclavos y libertos, halladas solamente en Caesarea, la capital oriental del reino (Matilla 1975, Gozalbes 1979). Se conoce una esclava de Juba II que murió a los 18 años ${ }^{17}$ y un esclavo de Ptolomeo de nombre Amarantus. ${ }^{18}$ Se conocen además nueve libertos de Juba II; ${ }^{19}$ ocho de Ptolomeo, entre los que hay cinco de nombre griego y tres de nombre latino, ${ }^{20}$ y una liberta de Urania, esposa de este último. ${ }^{21}$

${ }^{17}$ Ecloga regis Iuba, mima, CIL VI, 10110; Ricci 1994, Solin 2002: esp. 1385.

${ }^{18}$ CIL VIII, 21091.

${ }^{19}$ C. Iulius Aeschinis, CIL VIII, 9346; Aunigae, CIL VIII, 9348; Caelato, CIL VIII, 9349; Alypias, CIL VIII, 21085; Epapharae, CIL VIII, 21086; Iulia Fastila, CIL VIII, 21087; Iulia Phiale, CIL VIII, 21088; Philodemus, CIL VIII, 21089; Chius Aug. Iubatianus structor, CIL VI, 9046 es incluido como liberto de Juba por Ricci 1994: 193, no A.24; contra Solin 2002: 1386, que lo considera liberto de un Iubatus o Iuvatius; G. Iulius Iubae l(ibertus) Niceros cocus, Tran 2018.

${ }^{20}$ Paramonus l(ibertus) Ptol(emaeanus), CIL VI, 4078, se trata de un liberto dudoso, cf. Ricci 1994: 194, no A.32; Solin 2002: 1385-1386; Hymnus regis Ptolomaei l(ibertus), CIL VI, 20409, cf. Ricci, 1994: 193, no A.29; Solin 2002: 1385; Philocalus Pyladis, CIL VIII, 9351; Iulia Hyni, CIL VIII, 20409; Iuli Narcissus, Matilla, 1975: 129; C. Iulio, CIL VIII, 21093; C. Iulio, CIL VIII, 21094; Iulia Pieris, CIL VIII, 21095.

${ }^{21}$ Iulia Bodine, AE 1946, 102 = AE 1950, 209, Matilla 1975: 129. 
En el territorio occidental del reino no se ha hallado ninguna inscripción de época de la monarquía mauritana o del Alto Imperio que haga referencia a esclavos. En cuanto a los libertos, el más conocido y por contra el menos representativo de su estatus social fue el liberto regio Aedemón. ${ }^{22}$ Liberto de Ptolomeo, su nombre era C. Iulius Aedemon. ${ }^{23}$ Aedemon es una transcripción del

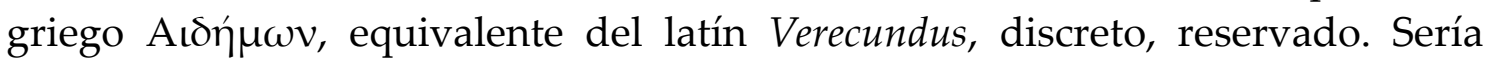
erróneo buscar en este nombre un origen griego $u$ oriental del personaje, ${ }^{24}$ dado que los nombres griegos de esclavos carecen de su significación étnica y además el nombre es del todo desconocido en el mundo greco-oriental (Lassère 1977: 435, Pereira 1981). Por lo que puede afirmarse su origen africano y que su nombre le haya sido dado mientras era esclavo. ${ }^{25}$ No sabemos si el resto de las familias de alto nivel económico del reino mauritano en esta época disponían de esclavos domésticos, lo que sería del todo habitual, ${ }^{26}$ pero no han dejado ningún rastro en la epigrafía.

Consideramos que la exportación se inició con Juba II, dado que este monarca fue el gran dinamizador del reino, siempre a favor de los intereses de Roma. Suetonio comenta cómo Augusto tenía en su corte niños esclavos de Mauritania y Siria, en sustitución de enanos (Aug. 83). La guerra de conquista de los años 40-42 d.C. y los periodos de conflictos con los indígenas, entre los años 118-122 d.C. y 171-178 d.C. son los momentos álgidos de la exportación de esclavos. En el s. III d.C., el Tarifario de Zarai, del 202 d.C. indica que el impuesto para los esclavos era de un denario y medio ${ }^{27}$ con lo que la exportación existía, pero debió de disminuir pues se redujeron los enfrentamientos (Rachet 1970, Gozalbes 1997: 196-197).

Para el periodo posterior a la conquista, conocemos un muy reducido número de libertos en las ciudades tingitanas. dos en Tingi, ${ }^{28}$ cuatro en Banasa, ${ }^{29}$ dos en Sala, ${ }^{30}$ y diez en Volubilis. ${ }^{31}$ Los estudios que han intentado esclarecer el

${ }^{22}$ Enc. Ber. sv. «Aedemon».

${ }^{23}$ I.A.M.lat., 448.

${ }^{24}$ Como cree Gozalbes 1979: 55. Cf. para la discusión sobre si los cognomina griegos denotan un estatuto servil o un origen greco-oriental, Santos Yanguas 1988.

${ }^{25}$ Gozalbes 1979: 55 plantea, en nuestra opinión de modo inconsistente, que el esclavo pudo ser comprado en Roma.

${ }^{26}$ Contra Gozalbes 1979: 65 para quien «El esclavismo, aparte de en la Corte, no tuvo el más mínimo desarrollo, ni siquiera entre las oligarquías ciudadanas de la época»; Cf. También Pereira 1981: 379-382.

${ }^{27}$ CIL VIII, 4508; Picard 1959: 94, Darmon, 1964, Guédon 2018: 179-186.

28 Antonius Hermes, I.A.M.lat., 2; Quintus Aelius Faustio, I.A.M.lat., 7; Caius Aurelius Herma, I.A.M.lat., 14; Valerius Eutychianus, I.A.M.lat., 33. Gozalbes 1979: 57, considera que podría existir otro liberto dudoso de nombre también Quintus Aelius Faustio.

${ }^{29}$ Lucius Antonius Charito, I.A.M.lat., 86; Marcus Terentius Primulus, I.A.M.lat., 88; Aelius Seimo, I.A.M.lat., 130; Cocceius, I.A.M.lat., 148.

30 Septimius Caruleianus, I.A.M.lat., 306; y Primigenius, I.A.M.lat., 318 que en nuestra opinión es dudoso.

${ }^{31}$ Sextus Iulius Epictetus, I.A.M.lat., 345; Lucius Caecilius Felix, I.A.M.lat., 352; Lucius Caecilius Vitalis, I.A.M.lat., 367; Aemilius Narcissus, I.A.M.lat., 429, 504; Aemilius Gandaro, I.A.M.lat., 429, 504; Aemilia 
porcentaje de esclavos y libertos en estas ciudades a partir de los restos epigráficos concluyen que entre las dos categorías sumaban un porcentaje alrededor del 10-20 \% (Marion 1948, 1950, 1960; Gozalbes 1979: 59-50). En el caso que considerásemos que un cognomen griego puede denotar un estatuto servil o un origen greco-oriental, ${ }^{32}$ deberíamos resaltar las siguientes evidencias. En primer lugar, se da una ausencia de esclavos con nombre griego en todas las poblaciones tingitanas; en segundo lugar, se da un predominio claro de libertos sobre esclavos e individuos libres con nombres griegos en Tingi, ciudad en la que casi todos los libertos portan un nombre griego; y en tercer lugar, en Volubilis se da un equilibrio entre libertos y libres con nombre griego, siendo la ciudad con un mayor número de individuos llevando nombres griegos (Santos Yanguas 1988: 863). En cuanto al medio rural, no existen estudios en el ámbito de la Tingitana que traten este tema.

En cuanto a la procedencia de los indígenas reducidos a la esclavitud, pudiéndose dar este fenómeno en todo el territorio, creemos que debió inscribirse especialmente al Sur de la provincia, donde se produjeron la mayor parte de los conflictos.

La visión que los romanos tenían de los esclavos africanos ha sido estudiada recientemente a partir de la poesía de Marcial y Juvenal. En cuanto a los esclavos de procedencia tingitana, los masiles aparecen solamente en Marcial, comentando que de su territorio se exportaban fieras para los espectáculos y esclavos, considerados de lujo. ${ }^{33}$ Los gétulos en Marcial presentan las mismas connotaciones que los masiles, mientras que en Juvenal son considerados como esclavos baratos y -si se nos permite la expresión- de gama baja. ${ }^{34}$ Los dos autores coinciden en la descripción de los rasgos físicos de los mauros, de piel oscura, con cabellos rizados y muy ágiles, ${ }^{35}$ tratándose de esclavos del todo comunes ya que la comida que se les daba para un mes resulta escasísima - jamón seco de mala calidad, un poco de atún, cebollas grilladas- y, por otro lado, las prostitutas que responden al nombre Maura son baratas y de mala reputación. ${ }^{36}$ Y en cuanto a la denominación «libio», que engloba diversos pueblos y es bastante imprecisa, es el término más corriente en Marcial para referirse a lo africano, incluyendo la importación a Roma de productos de lujo (marfil, maderas preciosas, o animales salvajes). Los esclavos que provienen de Libia son

Vrbana, I.A.M.lat., 429, 504; Marcus Valerius Antiochus, I.A.M.lat., 439; Caecilia Dativa, I.A.M.lat., 563; Caecilia Iusta, I.A.M.lat., 565; Iulius Fortunatus, I.A.M.lat., 601. Otros libertos dudosos comentados por Marion 1960 y Euzennat, 1971 son Iulius Nasser, I.A.M.lat., 491a; Iulius Narcissus, I.A.M.lat., 491a; Marcus Annius Heutycetis, I.A.M.lat., 491e; Marcus Valerius Hermes, I.A.M.lat., 491g; Iunius Aiax, I.A.M.lat., 491g; Claudius Sarpedo, I.A.M.lat., 492; Priamus, I.A.M.lat., 537; Iulius Felix, I.A.M.lat., 601.

32 Como hace Santos Yanguas 1988: 860.

${ }_{33}$ Mart. 10. 94; Garrido-Hory 1998: 923-924.

${ }^{34}$ Juv. 5. 53; 5. 59; Garrido-Hory 1998: 924. Cf. también Gascou 1970.

${ }^{35}$ Descripción coincidente con las que se conocen en las escenas de mosaicos que representan a bárbaros» norteafricanos; Neira 2004.

${ }^{36}$ Mart. 14. 196; Juv. 7. 120. 
obreros especializados. ${ }^{37}$ La conclusión a la que llegan los autores de este estudio es que, aunque los esclavos de procedencia africana representen solamente un pequeño porcentaje de la mano de obra servil en Roma, aparecen recurrentemente en las poesías de estos dos autores ya que son considerados esclavos de calidad, apareciendo siempre relacionados con casas ricas o con la Corte (Garrido-Hory 1998: 928). Con todo son víctimas de cierto racismo ${ }^{38}$ por parte de los autores clásicos al serles aplicados sistemáticamente los adjetivos ferreus, niger, obscurus o tristis, despectivos por varios motivos. La actitud despectiva hacia ellos no proviene del hecho de ser africanos sino del hecho de ser esclavos.

La exportación de esclavos mauritanos hacia Roma, mercado que conocemos por las fuentes epigráficas y literarias, estuvo, en definitiva, limitada a los esclavos de lujo de casas aristocráticas.

\section{LA PÚRPURA}

La púrpura fue durante toda la Antigüedad un símbolo de estatus social (Reinhold 1970). El uso de la púrpura como tinte fue una invención fenicia, siendo todavía durante el Imperio Romano la procedente de Tiro muy apreciada. Como herederos de los fenicios en el Mediterráneo Occidental, los cartagineses continuaron desarrollando esta actividad industrial (Wilson 2004). Plinio (Nat. 9. 38) describe el proceso industrial de fabricación de la púrpura: los moluscos más pequeños eran machacados, mientras que los de mayor tamaño eran abiertos con un instrumento cortante. El jugo extraído era depositado en contenedores donde se maceraba con sal durante tres días a una temperatura de $35^{\circ} \mathrm{C}$., después todavía necesitaba un tratamiento de diez días, siendo reducido por ebullición en recipientes de plomo con una aleación de antimonio (al $5 \%$ ) y un medio alcalino potásico, quizás obtenido de las cenizas de la madera que impedían la precipitación del color por oxidación, dado que éste solamente podía formarse con su exposición al aire. Las diferentes gamas de color obtenidas iban del rojo bermellón al violáceo y eran logradas mediante, por un lado, los diversos baños de la prenda a teñir en la tintura; o bien, por otro lado, mezclando diversas cantidades de los diferentes tintes obtenidos de las diferentes especies de moluscos. A pesar de la descripción de Plinio, existen dudas acerca de este proceso, como qué tipo de reductor se utilizaba, si se procedía con un proceso enzimático, el papel de la miel y el vinagre en la conservación de los tejidos teñidos con tinte purpúreo, etc. El producto resultante de este proceso eran los tejidos teñidos de este color, que se transformaban así en auténticas piezas de lujo (Jodin 1967; Fernández Uriel 1995, 1998: 74-81).

\footnotetext{
${ }^{37}$ Mart. 9. 101.

${ }^{38}$ El término «racismo» es de Garrido-Hory 1998: 928-931.
} 
A mediados del s. I d.C., la púrpura era un símbolo de estatus tan evidente que Calígula y Nerón, en relación con sus tendencias autocráticas, intentaron restringir su uso a la corte imperial y con esta excusa Calígula mandó asesinar a Ptolomeo de Mauritania, por vestir un manto purpúreo. ${ }^{39}$ Durante el principado de Nerón se restringió la venta y uso general de las dos categorías más elevadas de púrpura, la amethystina y la Tyria, reservadas para la corte. Nerón llegó a cerrar los comercios de estos productos de calidad en Roma y castigó una matrona que vestía vetia purpura con la confiscación de sus tierras. ${ }^{40}$ Con todo, no hay duda de que el uso de la púrpura tanto para uso privado como para uso oficial no hizo más que crecer durante el s. I d.C. (Dubois-Pelerin 2008: 230-232), como demuestran los comentarios de Plinio $^{41}$ y Marcial, ${ }^{42}$ así como las críticas moralizantes de Séneca ${ }^{43}$ y Quintiliano. ${ }^{44}$

Por lo que concierne a la púrpura mauritana, Plinio (Nat. 6. 202) indica que su explotación a gran escala solamente se dio a partir del reinado de Juba II: este monarca ordenó que fueran construidas instalaciones para su elaboración en unas islas situadas enfrente de la costa del territorio de los autololes, es decir Getulia (Besnier 1906: 282-283, Desanges 1964). De este pasaje podría deducirse que la fabricación de púrpura podría ser un monopolio real. Otra referencia a esta zona la ofrece Pomponio Mela (3.10) cuando afirma que nigrites y gaetuli, pueblos de vida nómada, no tenían problemas de subsistencia ya que obtenían púrpura de los murices, apreciada en todas partes. $\mathrm{Y}$ tenemos además la cita de Horacio (Carm. 2. 16. 35) según la que la púrpura mauritana era de gran calidad.

Esta área de producción, la más famosa en el extremo occidente y hasta hace poco considerada única, ha sido identificada con el islote de Mogador, delante de la población actual de Essaouira, en la costa central del actual Reino de Marruecos. El primero que intuyó que la ubicación de estas islas debía situarse en Mogador fue Vidal de la Blache (1902). Besnier (1904: 343, 1906: 282-283), contemporáneamente, las había identificado con el archipiélago de Madeira. Más tarde se produjeron ciertos hallazgos arqueológicos, publicados por Desjacques y Koerbelé (1955), que permitieron confirmar la hipótesis de Vidal de la Blache. Las causas propuestas para la fabricación de púrpura en Mogador son dos. En primer lugar, la existencia allí de la materia prima, es decir el molusco denominado según Plinio (Nat. 9. 37) Bucinum (Purpura Haemastoma) y el denominado purpura (Murex Trunculus). Y, en segundo lugar, el hecho que la fabricación de púrpura y la industria salazonera eran actividades estacionarias que podían fácilmente ser compaginadas. En verano y otoño podía llevarse a

\footnotetext{
39 Suet. Cal. 35. Este privilegio fue otorgado a la corte imperial en sentido amplio, incluyendo también al caballo de Calígula, Incitatus, según Suet. Cal. 55; Reinhold 1970: 49-50.

40 Suet. Nero 25, 32; Orosio 7. 7; Reinhold 1970: 50.

${ }^{41}$ Plin. Nat. 9. 53. 104; 9. 60 124-127; 9. 63. 137; 19. 22; 31. 123.

42 Mart. 1. 96. 7; 2. 57. 2; 5. 8; 6. 11; 8. 10.

${ }^{43}$ Sen. Ep. 16. 8; 76. 31; 90. 41; 94. 70; 114. 21; Sen. Thy. 343-344; Sen. Dial. 2. 13. 2.

${ }^{44}$ Quint. Inst. 1. 2. 6; 11. 1. 31; Reinhold 1970: 51.
} 
cabo la pesca y procesamiento de los pescados para la fabricación de salazones y garum; el resto del año podía llevarse a cabo la elaboración de la púrpura (Fernández Uriel 1995, 1998, 2009; Trakadas 2015: 12-13, 54-57).

La ciudad de Essauira parece haber sido en la Antigüedad una isla, que posteriormente fue unida al continente por la aportación de aluviones del río Ksob. Hoy en día, el islote de Mogador se halla en el centro de la bahía de Essauira, frente a esta ciudad. Mogador, nombre perpetuado en el morabito local de «Sidi Mogdul», podría significar «torre» o «fortaleza» en lengua fenicia occidental (Villaverde 2001: 190, nota 795). En época romana pudo haber sido conocido por su nombre helenístico «Cerné», ya que es con esta denominación como lo citan Plinio (Nat. 6. 199) o Zósimo (2. 33. 2). Es posible que este apelativo, que significa «cornamenta», provenga de la descripción figurada de la bahía, en forma de creciente lunar (Villaverde 2001: 190-192). Ambos nombres son traducidos al latín para formar la denominación que utiliza el Anónimo de Rávena, Turris Buconis (idem; Euzennat 1989: 107-109). Los alrededores de Essauira estaban, como en la actualidad, formados por arenales improductivos desde el punto de vista agrícola y quizás solamente en la zona más inmediata a la ribera del río se hubiera podido producir un cultivo de huerta. Otros recursos de la ciudad podrían haber sido la explotación forestal de una comarca de pequeños montes que se halla a unos $20 \mathrm{~km}$ hacia el Este; la venta de la sandáraca; o el comercio, pues la bahía de Esauira era el mejor fondeadero natural del Sur del litoral atlántico marroquí y además la isla de Mogador permitía a los comerciantes no ser atacados directamente por los indígenas (Mederos y Escribano 2002, López Pardo 2002, Tejera y Chávez 2004).

La cronología general de Mogador abarcaría desde el s. VII a.C. al V-VI d.C. Fue sin duda un activo lugar de desembarco, utilizado por los fenicios de occidente en época arcaica, seguramente gadeiritas, habiéndose hallado mobiliario de los siglos VII-VI a.C. (Habibi 2001, Marzoli y Khayari 2009, López Pardo y Mederos 2008, López Pardo et alii 2011). Pero al tratarse de un lugar falto de estructuras arquitectónicas fijas de esa cronología, dificulta el proponer hipótesis sobre la existencia de un hábitat estable, debiéndose considerar entonces como «una base marítima de transacción comercial habitada» (Ramon 1995: 96). Posteriormente, se dio una larga fase de abandono hasta el s. I a.C., produciéndose otro abandono del lugar en el s. I d.C. (Jodin 1957: 19). Se ha sugerido que la causa del abandono fuera la poca rentabilidad de estas producciones en el confín de la provincia durante la época altoimperial, cuando ésta intensifica su actividad agraria (Villaverde 2001: 192), aunque parecería que esta hipótesis entrara en contradicción con el testimonio de diversos autores romanos que destacan en el s. I d.C. la calidad de la púrpura de Getulia. ${ }^{45}$ En el s. III d.C., en época severiana, se reanuda la producción de púrpura de Mogador,

${ }_{45}$ Plin. Nat. 9, 127; Hor. Carm. 2. 16. 35; Ep. 2. 2. 181; Ov. Ars. 3. 170; Juv. 8. 101; 9.19. 
aunque la consolidación de su población no se dará hasta el s. V d.C. (Jodin 1957: 19, Villaverde 2001: 192).

Jodin afirmó que se trataba esencialmente de un complejo industrial de dos cetariae, asociadas a una villa, que inició su producción de púrpura con Juba II. Se hallaron varias docenas de anzuelos, testimonio de los millares que debieron ser utilizados; gran número de restos de fauna marina de todo tipo (bivalvos, ostras, mejillones, entre otros); se construyeron una red de cisternas y canalizaciones para solucionar el abastecimiento de agua, lo que permite pensar en unas instalaciones industriales de cierta importancia; se construyeron depósitos similares a los utilizados en las factorías de salazones; se construyeron también hipocaustos, como hemos visto, necesarios para el proceso productivo de la púrpura; no se hallaron los depósitos de plomo para macerar la tintura, pero sí varios $\mathrm{kg}$ de este material (Jodin 1967: 256-257). Las excavaciones del INSAP y el DAI-Madrid entre 2006 y 2008 han precisado las cronologías: la ocupación tardorepublicana puede datarse con anterioridad al s. I a.C., la villa y las cetariae están ocupadas y produciendo púrpura en el s. II d.C. (Marzoli y Khayari 2009, 2010).

Algunos investigadores afirmaban que la producción de púrpura también pudo producirse en la zona del Estrecho, pero sus argumentos se reducían a atestiguar la presencia de los múrices en sus costas y en algunas excavaciones arqueológicas, así como la posibilidad de rastrear esta producción en la toponimia, sin precisar si realmente el uso que se dio a estos restos era el de la fabricación de púrpura (Ponsich 1988: 54, García Vargas 2004, Fernández Uriel 1998: 80, nota 40). López Pardo se manifestó contrario a esta hipótesis: en primer lugar, consideraba que solamente en Getulia podían encontrarse moluscos en suficiente cantidad para producir púrpura con el método antes expuesto y, por tanto, solamente allí la industria de la púrpura sería subsidiaria de la de salazones de una forma mecánica. Proponía además tener en cuenta la hipótesis de Gattefossé según la cual la púrpura gétula podría haber sido fabricada, al menos en parte, a partir de un liquen, la urchilla de mar (Rocella tinctoria), que se halla en Mogador y en la costa cercana (Gattefossé 1957; López Pardo 1987: 196, 1992). En segundo lugar, creía que no puede afirmarse con seguridad que en Mogador existiera una industria de fabricación de púrpura dado no se habían hallado las conchas de estos moluscos en ninguna parte de la isla (López Pardo 1987: 194196), solamente en la costa cercana (Desajacques y Koerbele 1955: 198-199); de manera que siendo la púrpura sin duda una producción de Getulia, no podría afirmarse que hubiera sido producida precisamente en Mogador. Y en tercer lugar, era de la opinión que las fuentes clásicas no ofrecían ningún dato relativo a una producción de púrpura en la zona del Estrecho: ninguno de los autores que tratan la producción de salazones allí aluden a la producción de púrpura, ni tan solo Pomponio Mela, buen conocedor de la zona (López Pardo 1987: 194). Pero los argumentos ex silentio resultan engañosos y en 2008 se descubrió una factoría de salazones y púrpura en Metrouna, al este de Septem Fratres, en la 
desembocadura del río Martil y cerca del ya conocido yacimiento de Sidi Abdeslam del Behar. Se han detectado en Metrouna varios concheros destinados al machacado de Heraplex trunculus para obtener el preciado colorante con lo que se confirma que la cetaria fabricó púrpura; estaría activa entre 75 y 150 d.C. (Bernal et alii 2104, Trakadas 2015: 27-29, 2018: 447). Por otro lado, en la cetaria de la Plaza de África de Septem Frates se han hallado indicios de la fabricación de púrpura (unas 100 conchas de Murex/Hexaplex trunculus machacadas y con evidencias de haber sido calentadas) en cronologías del s. V-VI d.C. (Trakadas 2015: 32-35, 2018: 233)

\section{CONCLUSIONES}

Si realizamos una lectura crítica de las fuentes disponibles, concluimos que la explotación de estos productos del reino de Mauritania, luxuria mauretaniae, no pueden ser la causa de la conquista del reino por Calígula (40 d.C.). En primer lugar, porque la datación del inicio de la explotación de estos productos es coincidente: Juba II, que como monarca vasallo permitió el aprovechamiento sin medida de los recursos naturales del reino que gobernaba por voluntad exclusiva de Augusto. $\mathrm{Y}$ en segundo lugar, porque no era en absoluto necesaria, como vemos, la transformación del reino en provincia para llevar a cabo esta explotación pues funcionaba perfectamente, como mínimo, desde la entronización de Juba II (25 a.C.): formaba parte de facto del imperio. Las cronologías de inicio y fin de la explotación de estos productos son similares. La madera de sabina de Cartagena (citrus) era explotada por los comerciantes romanos desde época de Juba II y se produjo su agotamiento en el s. II d.C. El marfil se exportaba ya desde época cartaginesa, pero fue con Juba II cuando su exportación aumentó significativamente, agotándose en época Flavia en esa zona. En cuanto a los esclavos, la primera referencia conocida es de época de Augusto; reconocemos que las fuentes son poco explícitas en este punto e inferimos que se exportarían en mayor número en épocas de conflictos con los indígenas. La púrpura empezó a producirse en Mauritania a gran escala bajo Juba II, no agotándose nunca este recurso.

\section{BIBLIOGRAFÍA}

Amela VAlVerde, L. (2012), «La situación de Mauritania a finales del Segundo Triunvirato e inicios del principado de Augusto», Gerión 30, 149-167.

AnTOINE, M. (1931), «L'Elephas africanus au Maroc», Bulletin de la Société de préhistoire du Maroc 5, 69.

AubeT, M. E. (1994), Tiro y las colonias fenicios de Occidente, Barcelona, Ediciones Bellaterra.

BEAUDET, G. (1971), «Le Quaternaire marocain: état des études», Revue de Géographie du Maroc 20, 6. 
BERNAL-CASASOlA. D. et alii (2014), «De la producción de púrpura getúlica. Arqueomalacología en la cetaria altoimperial de Metrouna», en Purpureae vestes, I., Production and Trade of Textiles and Dyes in the Roman Empire and Neighbouring Regions. Producción y comercio de textiles y tintes en el imperio romano y regiones cercanas, Alfaro C., Tellenbach M., Ortiz J. (coords.), Valencia, Universitat de València, 175-188.

BERNARD, G. (2018), Nec Plus Ultra. L'Extrême Occident méditerranéen dans l'espace politique romain (218 av. J.-C. - 305 apr. J.-C.). Madrid, Casa de Velázquez.

BESNIER, M. (1904), «Géographie ancienne du Maroc (Maurétanie Tingitane)», Archives Marocaines, 301-365.

BESNIER, M. (1906), «La géographie économique du Maroc dans l'Antiquité», Archives Marocaines 7, 271-295.

BIFFI, N. (1999), L'Africa di Strabone. Libro XVII della Geografia. Introduzione, traduzione e comento, Bari, Modugno.

CARCoPINO, J. (1943), Le Maroc Antique, Paris, Gallimard.

CHEDDAD, A. (2002), «Recherches de géographie historique: à propos du Mont Atlas», en L'Africa romana. XIV Convegno Internazionale di Studi. Lo spazio marittimo del Mediterraneo Occidentale: geografia storica ed economia. Sassari, 7-10 dicembre 2000, Khanoussi, M., Ruggeri, P. y Vismara, C. (eds.), Roma, Carocci, vol. 3, 2163-2176.

CIMMA, M. R. (1976), Reges socii et amici populi romani, Milano, Giuffrè.

Coltelloni-TranNOY, M. (1988-1989), «Le monnayage des rois Juba II et Ptolomée de Maurétanie: image d'une adhésion réitérée à la politique romaine», Karthago XXII, 45-53.

Coltelloni-Trannoy, M. (1997), Le royaume de Maurétanie sous Juba II et Ptolémée, Paris, Éds. du C.N.R.S.

DALBY, A. (2000), Empire of Pleasures: Luxury and Indulgence in the Roman world, LondonNew York, Routledge.

DARMON, J.-P. (1964), «Note sur le Tarif de Zaraï», Les Cahiers de la Tunisie 47-48, 7-23.

Delgado Delgado, J. A. (2011), «Mogador (Essaoutia. Marruecos), un commercium Romano de citrum en la periferia sudoccidental del imperio», Athenaeum 96, 155174.

De Martino, F. (1985), Historia Económica de la Roma Antigua, Madrid, Akal.

DESANGES, J. (1964), «Les territoires gétules de Juba II», Revue des Études Anciennes 66, 3347.

DesjaCQues, J. y P. Koerbele (1955), «Mogador et les Iles Purpuraires», Hespéris 42, $193-$ 202.

Di VITA-ÉVRARD, G. (1992), «La dédicace des Horrea de Tubusuctu et l'ère de la province dans les Maurétanies», en L'Africa romana. Atti del IX Convegno di studio, Nuoro, 1315 dicembre 1991, Mastino. A. (a cura di), Sassari, Publicazioni del Dipartimento di Storia dell'Università di Sassari, Gallizi, vol. 2, 843-864.

DI VITA-ÉVRARD, G. (1994), «L'ère de Maurétanie: une nouvelle attestation», en L'Africa romana. Atti del X Convegno di studio. Oristano, 11-13 dicembre 1992, Mastino, A., Ruggeri, P. (a cura di), Sassari, Pubblicazione del Dipartimento di Storia dell'Università degli Studi di Sassari, vol. 3, 1061-1070.

Dubois-Pelerin, E. (2008), Le luxe privé à Rome et en Italie au 1er siècle ap. J.-C., Naples, Centre Jean Bérard. 
EUZENNAT, M. (1971), «Grecs et orientaux en Maurétanie Tingitane», Antiquités africaines $5,161-178$.

EuZENNAT, M. (1989), «Remarques sur la description de la Maurétanie Tingitane dans Pline», H.N., V, 2-18. Antiquités africaines 25, 95-109.

FAUR, J.-C. (1973), «Caligula et la Maurétanie: La fin de Ptolomée», Klio. Beitrage zur alten Geschichte 55, 249-271.

FERNÁNDEZ URIEL, P. (1995), «La púrpura en el Mediterráneo Occidental», en II Congreso Internacional «El Estrecho de Gibraltar»(Ceuta, 1990), Ripoll Perelló, E. et alii., Madrid, UNED, 309-327.

FERNÁNDEZ URIEL, P. (1998), «Melilla en el comercio del Mediterráneo: miel, sal y púrpura», Melilla y su entorno en la antigüedad, Melilla, UNED, 53-87.

FERNÁNDEZ URIEL, P. (2009), Púrpura. Del mercado al poder, Madrid, UNED, 232-238.

GARCÍA VARGAS, E. (2004), «Las pesquerías de la Bética durante el Imperio Romano y la producción de púrpura», en Purpureae vestes. Actas del I Symposium Internacional sobre textiles y tintes del mediterráneo en época romana, Alfaro Giner, C., Peter Wild, J., Costa Ribas, B. (coord.), Eivissa, Universitat de València-Consell Insular d'Eivissa, 219-235.

GARRIDO-HORY, M. (1998), «Les esclaves africains dans la poésie réaliste», en L'Africa romana. Atti del XII Convegno di studio, Olbia, 12-15 dicembre 1996, Khanoussi, M., Ruggeri, P. y Vismara, C. (eds.), Sassari, Editrice democratica sarda, vol. 2, 921-935.

GASCOU, J. (1970), «Le cognomen gaetulus, gaetulicus en Afrique romaine», MEFRA 82, 723-736.

GATTEFOSSE, J. (1957), «La pourpre gétule invention du roi Juba de Maurétanie», Hespéris 44, 329-334.

GHAZI-BEN MAÏsSA, H. (1995), «Encore et toujours sur la mort de Ptolémée, le roi amazigh de Maurétanie», Hespéris-Tamuda 33, 21-37.

GozAlBES CRAVIOTO, E. (1979), «Consideraciones sobre la esclavitud en las provincias romanas de Mauritania», Cahiers de Tunisie 107-108, 35-67.

GozAlbes CRAvioto, E. (1988), «Los elefantes de Septem Frates», Cuadernos del Archivo Municipal de Ceuta 2, 3-12.

GOZALBES CRAVIOTO, E. (1988), «La conquista romana de Mauretania», Studi magrebini 20, 1-43.

GozAlbes CRAVIOTO, E. (1995), «La descripción de la Mauritania Tingitana en Pomponio Mela», en II Congreso Internacional «El Estrecho de Gibraltar» (Ceuta, 1990), Ripoll Perelló, E., Ladero Quesada, M. F., (coord.), Madrid, UNED, 259-265.

Gozalbes Cravioto, E. (1997), Economía de la Mauritania Tingitana (Siglos I a.C. - II d.C.), Ceuta, Instituto de Estudios Ceutíes.

GselL, S. (1920), Histoire Ancienne de l'Afrique du Nord, 1913-1928 [1972], vol. 1., Paris, Hachette.

GuASTELla, G. (1994), Gaio Svetonio Tranquilo. La vita di Caligola, Roma, La Nuova Italia Scientifica.

GuEY, J. (1947), «Les éléphants de Caracalla», Revue des Études Anciennes 49, 248-273.

GuEDON, S. (2018), La frontière romaine de l'Africa sous le Haut-Empire, Madrid, Casa de Velázquez.

HABIBI, M. (2001), «Le comptoir phénicien de Mogador: Approche chronologique et céramique à engobe rouge», en Actes des 1ères Journées Nationales d'Archéologie et du Patrimoine, Rabat, Société Marocaine d'Archéologie et du Patrimoine, 53-63. 
Hamdoune, CH. (2009), «Mons Africae uel fabulosissimus Atlas: l'Atlas de Pline l'Ancien", Latomus 68, 101-116.

HARRISON, R. J. Y A. GILMAN (1977), «Trade in the second and third milenia B.C. between Maghreb, Iberia», en Ancient Europe, the Mediterranean. Studies presented in honour of Hugh Hencken, Markotic, V. (ed.), Warminster, Aris and Phillips.

HoffmanN, M. (1959), «Ptolemaios von Mauretanien», en Paulys Real Enciclopädie der Classichen Altertumwissenschaft, Wissowa, G., Kroll, W., Mittelhaus, K., Ziegler, K. (eds.), Stuttgart, Alfred Druckenmüller Verlag, vol. 23.2, 1768-1787.

JODIN, A. (1957), «Note préliminaire sur l'établissement pré-romain de Mogador (campagnes 1956-1957)», Bulletin d'Archéologie Marocaine 2, 9-40,

JoDiN, A. (1967), Les établissements du roi Juba II aux Illes Purpuraires (Mogador), Tanger, Editorial Tanger.

JODIN, A. (1970a), «L'exploitation forestière du Maroc antique», en Actes du 93e Congrès Nationale des Sociétés Savantes. Tours. 1968. Section Archéologie, Paris, Bibliothèque nationale, 413-422.

JoDin, A. (1970b), «L'éléphant dans le Maroc antique», en Actes du 92e Congrès Nationale des Sociétés Savantes. Strasbourg et Colmar. 1967. Section d'Archéologie, Paris, Bibliothèque nationale, 51-64.

KeLLER, O. (1904), Kulturgeschichtliches aus der Tierwelt, Prag.

KelLer, O. (1909-1914), Die antike Tierwelt, 2 vol., Leipzig, Wilhelm Engelmann Verlag.

KotUla, T. (1964), «Encore sur la mort de Ptolomée roi de Maurétanie», Archeologia_15, 76-92.

LASSERE, J.-M. (1977), Ubique Populus. Peuplement et mouvements de population dans l'Afrique romaine de la chute de Carthage à la fin de la dynastie des Sévères (146 a. C.-235 p. C.), Paris, CNRS.

LASSERE, J. M. (1995), «Productions et exportations africaines. Les limites du témoignage de l'épigraphie lapidaire», en VIe Colloque international sur l'histoire et l'archéologie de l'Afrique du Nord (Pau, octobre 1993 - 118e congrès). L'Afrique du Nord Antique et Médievale. Productions et exportations africaines. Actualités archéologiques, Trousset, P., Paris, CTHS, 39-45.

LENOIR, M. (2001), «La piste égyptienne de Marc-Antoine, la cagnotte dilapidée et l'empereur psychopathe. L'élimination de Ptolémée, roi de Maurétanie», en Vbique amici. Mélanges offerts à Jean-Marie Lassère, Hamdoune, C. (ed.), Montpellier, Centre d'études et de recherches sur les civilisations antiques de la méditerranée, 117-127.

LEVEAU, P. (1984a), «La fin du royaume maure et les origines de la province romaine de Maurétanie Césarienne», Bulletin du Comité des Travaux Historiques et scientifiques, 17B, 313-321.

LEVEAU, P. (1984b), Caesarea de Maurétanie. Une ville romaine et ses campagnes, Rome, Ecole Française de Rome.

LóPez GonZÁLEZ, G. (2001), Los árboles y arbustos de la Península Ibérica e Islas Baleares, tomo I, Barcelona, Mundiprensa.

LÓPez PARDO, F. (1987), Mauritania Tingitana: de mercado colonial púnico a provincia periférica romana, Madrid, Universidad Complutense de Madrid.

LÓPEZ PARDO, F. (1992), «Mogador: "Factoría extrema” y la cuestión del comercio fenicio en la costa atlántica africana», en Afrique du Nord Antique et Médievale. Spectacles, vie portuaire, religions. Actes du ve Congrès International sur l'Histoire et l'Archéologie de l'Afrique du nord, dans le cadre du 115e Congrés Nationale des Sociétés Savantes 
(Avignon, 9-13 Avril 1990), Duval, N., Rebuffat, R., Lepelley, Cl., Fantar, M. (ed.), Paris, CTHS, 277-296.

LÓPEZ PARDO, F. (2002), «Sandáraca, el ámbar de los dioses, en las costas de la factoría fenicia de Mogador/Kerné (Marruecos Atlántico)», Akros 1, 48-53.

LÓPEZ PARDO, F. y A. MEDEROS (2008), La factoría fenicia de la isla de Mogador y los pueblos del Atlas, Tenerife, Museo arqueológico de Tenerife.

LÓPEZ PARDO, F. et alii (2011), «Prospección arqueológica de Mogador y su territorio continental inmediato, Campaña de 2000», Canarias Arqueológica: arqueologíabioantropología 19, 109-148.

MARION, J. (1948), «Note sur le peuplement de Tanger à l'époque romaine», Hespéris 35, 125-149.

MARION, J. (1950), «Note sur le peuplement de Sala à l'époque romaine», Hespéris 37, 399427.

MARION, J. (1950), «Note sur le peuplement de Banasa à l'époque romaine», Hespéris 37, $57-180$

MARION, J. (1960), «La population de Volubilis à l'époque romaine», Bulletin d'Archéologie Marocaine 4, 133-187.

MARZOLI, M. Y A. El KHAYARI (2009), «Mogador (Essaouira, Marokko). Vorbericht über die Kampagnen 2006 und 2007 mit einem Beitrag von H. Brückner und J. Lucas», Madrider Mitteilungen L, 80-117.

MARZOLI, M. Y A. EL KHAYARI (2010), «Vorbericht Mogador (Marokko) 2008», Madrider Mitteilungen LI, 61-108.

MAtILlA, E. (1975), «Esclavitud en la Mauritania Cesariense», Revista internacional de sociología 13-14, 109-136.

Mederos, A. y G. Escribano (2002), Fenicios, púnicos y romanos. Descubrimiento y poblamiento en las Islas Canarias, Madrid, Gobierno de Canarias, Dirección General de Patrimonio Histórico.

MAZARD, J. (1955), Corpus Nummorum Numidiae Mauretaniaeque, Paris, Gouvernement Général de l'Algérie.

NEIRA, M. L. (2004), «La imagen del "otro": Representaciones de "bárbaros" en la musivaria romana», en L'Africa romana. XV Convegno Internazionale di Studi. Ai confini dell'Impero: contatti, scambi, conflitti. Tozeur, 12-15 dicembre 2002, Khanoussi, M., Ruggeri, P., Vismara, C. (ed.), Roma, Carocci, vol. 1, 877-894.

PAGNOUX, C. (2017), «Investigating the introduction of citrus fruit in the Western Mediterranean according to ancient Greek and Latin texts», en AGRUMED: Archaeology and history of citrus fruit in the Mediterranean: Acclimatization, diversifications, uses, Zech-Matterne,V., Fiorentino, G. (eds.), Naples, Publications du Centre Jean Bérard.

PereiRA, G. (1981), «El número de esclavos en las provincias romanas del Mediterráneo, en el Imperio», Klio 63.2, 373-399.

PÉREZ GonZÁlEZ, J. (2014), «La venta de perlas en la ciudad de Roma durante el Alto Imperio», Espacio, tiempo y forma. Serie II Historia Antigua 27, 267-289.

PÉREZ GONZÁLEZ, J. (2019), «How Roman Sumptuary Specialists Called Themselves: A Corpus-Based Study, Latomus 78.4, 996-1037.

PICARD, G.-CH. (1959), La civilisation de l'Afrique romaine, Paris, Plon.

PONSICH, M. (1988), Aceite de oliva y salazones de pescado. Factores geoeconómicos de Bética y Tingitania, Madrid, Editorial Complutense. 
PONS Pujol, Ll. (1997), «Volubilis i els bona vacantia: una síntesi», Pyrenae 28, 133-149.

PONS PUJOL, LL. (2008), «Consecuencias jurídicas de una masacre: Volubilis, 40/41 d.C.», IVRA, 157-174.

PONS PujOL, LL. (2009), La economía de la Mauretania Tingitana (s. I-III d.C.). Aceite, vino y salazones, Barcelona, Publicacions de l'Universitat de Barcelona.

PONS PUJOL, LL. (2014), «Umbonius Silio y el abastecimiento militar de la Tingitana (Dión Casio, LX, 24.5)», en La guerre dans l'Afrique romaine sous le Haut-Empire, ColtelloniTrannoy, M., Le Bohec, Y. (eds.), Paris, CTHS, 167-176.

PONS PujOL, LL. (2020), «La legación de M. Licinius Crassus Frugi en Mauretania (CIL VI 31721)», en Ex Baetica Romam. Homenaje a José Remesal Rodríguez, Revilla Calvo, V., Aguilera Martín, A., Pons Pujol, Ll., García Sánchez, M. (eds.), Barcelona, Edicions i Publicacions UB, 389-409.

RACHET, M. (1970), Rome et les Berbères, Bruxelles, Latomus.

RAMON, J. (1995), Las ánforas fenicio-púnicas del Mediterráneo central y occidental, Barcelona, Publicacions de la Universitat de Barcelona.

ReINHOlD, M. (1970), History of Purple as a Status Symbol in Antiquity, Bruxelles, Latomus.

RICCI, C (1994), «Africani a Roma. Testimonianze africane di età imperiale di personaggi provenienti dal Nordafrica», Antiquités africaines 30, 189-207.

Rostovzev, M. (1933[1992]), Storia Economica e Sociale dell'Impero Romano, Firenze, La Nuova Italia Editrice.

SANTOS YANGUAS, J. (1988), «Nombres griegos en la epigrafía romana de la Bética y la Mauritania Tingitana», en I Congreso Internacional «El Estrecho de Gibraltar» (Ceuta, 1987), Ripoll Perelló, E. (ed.), Madrid, UNED, vol. 1, 855-864.

SCHETTINO, M. T. (2003), «La Mauretania dal tardo ellenismo alla provincializazione», en Gli stati territoriali nel mondo antico, Bearzot, C., Landucci Gattinoni, F., Zecchini, G. (a cura di), Milano, Vita e Pensiero, 289-316.

SigmAN, M. C. (1977), «The Romans and the Indigenous Tribes of Mauritania Tingitana», en Historia. Zeitschrift für Alte Gesichte. Revue d'Histoire Ancienne. Journal of Ancient History. Rivista di Storia Antica 26, 415-439.

SIRAGO, V. A. (1996), «Il contributo di Giuba II alla conoscenza dell'Africa», en L'Africa romana. Atti del XI Convegno di studio, Carthagine, 15-18 dicembre 1994, Ozieri, Il Torchietto, vol. 1, 303-317.

SIRAJ, A. (1995), L'image de la Tingitane. L'historiographie arabe médievale et l'antiquité nordafricane, Rome, EFR.

SoliN, H. (2002), «Appunti sulla presenza di Africani a Roma, en L'Africa romana. XIV Convegno Internazionale di Studi. Lo spazio marittimo del Mediterraneo Occidentale: geografia storica ed economia. Sassari, 7-10 dicembre 2000, Khanoussi, M., Ruggeri, P., Vismara, C. (ed.), Roma, Carocci, vol. 2, 1381-1386.

SOUVILle, G. (1983), «Réflexions sur les relations entre l'Afrique et la Péninsule Ibérique aux temps Préhistoriques et Protohistoriques», en Homenaje al Prof. Martín Almagro Basch, Madrid, Ministerio de Cultura, 407-415.

Tejera Gaspar, A. y CHÁvez Álvarez, M. E. (2004), «La púrpura getúlica de la Mauretania Tingitana», en Purpureae vestes. Actas del I Symposium Internacional sobre textiles y tintes del mediterráneo en época romana, Alfaro Giner, C., Wild, J. P., Costa Ribas, B. (coords.), Valencia, Universitat de València: Consell Insular d'Eivissa i Formentera, 237-240. 
TRAN, N. (2013), «Le cuisinier G. Iulius Niceros et la domesticité royale de Maurétanie», Zeitschrift für Papyrologie und Epigraphik 187, 310-316.

TRAKADAS, A. (2015), Fish-salting in the Northwest Maghreb in Antiquity. A Gazetteer of Sites and Resources, Oxford, Archaeopress Archaeology.

TRAKADAS, A. (2018), In Mauretaniae maritimis. Marine Resource Exploitation in a Roman North African Province, Stuttgart, Steiner Verlag.

VIDAL DE LA BlACHE, P. (1902), «Les purpuraires du roi Juba», Mélanges Perrot, Paris, Librairie Thorin et Fils, 325-329.

Villaverde, N. (2001), Tingitana en la Antigüedad tardía (siglos III-VII). Autoctonía y romanidad en el extremo occidente mediterráneo, Madrid, Real Academia de la Historia.

WILSON, A. (2004), «Archaeological Evidence for textile production and dyeing in Roman North Africa», en Purpureae vestes. Actas del I Symposium Internacional sobre textiles y tintes del mediterráneo en época romana, Alfaro Giner, C., Wild, J. P., Costa Ribas, B. (coords.), Valencia, Universitat de València: Consell Insular d'Eivissa i Formentera, 155-164. 